\title{
CORRECTION
}

\section{Correction to: Paradox effects of binge drinking on response inhibition processes depending on mental workload}

\author{
Ann-Kathrin Stock ${ }^{1} \cdot$ Lea Riegler $^{1} \cdot$ Witold X. Chmielewski $^{1} \cdot$ Christian Beste $^{1}$
}

Published online: 7 January 2022

(c) Springer-Verlag GmbH Germany, part of Springer Nature 2022

\section{Correction to: Arch Toxicol (2016) 90:1429-1436 https://doi.org/10.1007/s00204-015-1565-y}

In the description of the experimental paradigm, we incorrectly stated that the experimental blocks $\mathrm{A}$ and $\mathrm{B}$ were presented in a counterbalanced order (as we had done in a previous publication using this paradigm, compare https:// doi.org/10.1016/j.neuroimage.2015.02.060). However, block B was actually always presented before block A in the current study. As block order was not investigated in this study, the reported results and values, as well as the interpretation of the findings remain unchanged.

Also, the y-axes of Fig. 2 are incorrectly labelled as "rate of false alarms (\%)", when "number of false alarms" would have been the correct label.
Publisher's Note Springer Nature remains neutral with regard to jurisdictional claims in published maps and institutional affiliations.

The original article can be found online at https://doi.org/10.1007/ s00204-015-1565-y.

Christian Beste

christian.beste@uniklinikum-dresden.de

Ann-Kathrin Stock

ann-kathrin.stock@uniklinikum-dresden.de

1 Cognitive Neurophysiology, Department of Child and Adolescent Psychiatry, Faculty of Medicine of the TU

Dresden, Schubertstrasse 42, 01309 Dresden, Germany 